\title{
Understanding the Artificial
}

\author{
H. Jaap van den Herik • Maarten Lamers • \\ Fons Verbeek
}

Published online: 23 February 2011

(C) The Author(s) 2011. This article is published with open access at Springerlink.com

The state of the art of robotics is currently hotly debated by two sides providing us with arguments from different perspectives. The technology-driven side tells that the world is driven and run by technological developments, and that robots are here for further enhancements and new applications. It means no less than that technology dictates the governance. The society-driven side opines that the world is driven and run by social aspects. The society (of human beings) dictates the governance. For instance, legislative drafting comes from humans and is not imposed by technological possibilities. Remarkably enough, both sides claim that they understand the artificial. For the technology-driven side, it is a reason to have robots involved in their procedures and developments; for the society-driven side, the reverse is true, they believe that further developing of the artificial should be initiated by human beings.

A prevailing question is: which position does frontranked research claim to possess at this moment? It is difficult to pinpoint such a position quite precisely. Clearly, the technology side would like to measure the progress by robotics by means of the 3 Is, being Interaction, Intelligence, and Imagination, whereas the society side takes as its measures the 3 Ss, being Safety, Security, and Supervision. No wonder that the outcome of the debate in 2011 is to be assessed as "undecided".

For a better understanding of the artificial, we would like to return a quarter of a century and then analyze the devel-

H.J. van den Herik ( $\square)$

Tilburg Center for Cognition and Communication (TiCC),

Tilburg University, Tilburg, The Netherlands

e-mail: jaapvandenherik@gmail.com

M. Lamers · F. Verbeek

Leiden Institute of Advanced Computer Science (LIACS), Leiden University, Leiden, The Netherlands opment and try to extrapolate the results into the future, say another twenty-five years. In the early years (from 1985 onwards) the building of robots was dominated by implementing knowledge (mostly domain knowledge), heuristics (for acting adequately), and search (for finding a way in a variety of labyrinths). Let us admit that thorough analysis proves that these three items have developed quite considerably and satisfiably over the last 25 years.

Leaving the past behind us and entering the current timeframe, let us attempt to characterize the period 2010-2015. We then observe that the robotic behavior is guided by (1) machine learning, (2) adaptivity, and (3) autonomy. For sure, all three items will be enhanced in the coming five years. However, that will not be the end of the development. It is even more likely that in 2015 the debate between the two sides has not been resolved either. Robots are companions showing to a large extent adaptivity to our wishes (and to non-verbal gestures), but not in an independent way (so, there is only some restricted autonomy, namely for "easy" decisions). Up to this point, one need not be clairvoyant to predict that this will be the logical development up to 2015 .

For the ten years thereafter, the prediction is more speculative. According to your Editors, we will live in a world where robots play an important part (definitively not a major part), and where the following three issues are implemented as attributes or even properties: (1) connectedness, (2) identity, and (3) reciprocity. Robots will be connected to each other and to human beings by their communication channels. It is good for safety and security, in particular if the robots act as car drivers, as supervisors (i.e., as intelligent sensor systems) and health-care workers. Robots then will have been assigned an identity, which is more than an IP address. The identity informs the humans who (which robot) is communicating with the human being and what role the robot fulfils (supervising, supportive, potential opponent). 
Subsequently, a supervising robot is endowed with a "polite feeling" of reciprocity, in that it will inform a human being on his (her) being captured (e.g., photographed) by the system when he/she was in the football stadium (or in another public building). Admittedly, real reciprocity has to do with mutual understanding; and even in 2025 we believe that a robot will not show any form of real understanding. In 15 years we will see what comes true of this speculation.

Yet, there is more, and therefore we continue to speculate on the following ten years, too. In the period from 2025 to 2035 we predict that research will (attempt to) develop robots that can socialize, that may have empathy, and that have (self)-consciousness. At that time and assuming the predictions come true, the technology debate will have been resolved. Your Editors then may have to conclude that understanding the artificial meanwhile has evoked the challenging task of understanding the artificial by the artificial. This, in our opinion, is an avoidable truth. We are willing to admit that its prediction for 2035 is optimistic, but the course of the development is certain.

The seven papers in this special issue "understanding the artificial" inform us in a scientific way on this development. Each paper does so with its own characteristics and due emphasis on different aspects. Below we briefly provide an overview of the contents of the papers.

Looking forward to a "Robotic Society"? Notions of Future Human-Robot Relationships by Astrid Weiss, Judith Igelsböck, Daniele Wurhofer, and Manfred Tscheligi reports on an explorative investigation. The notions of future human-robot relationships are specified. By means of 58 indepth interviews (52 novices and 6 experts), data was gathered on four notions: (1) the quality of life, health, and security, (2) the working conditions and employment, (3) education, and (4) cultural context. Five key aspects of the future 'robotic society' are then identified: (1) replacement, (2) competition, (3) safety and supervision, (4) increasing productivity, and (5) cost and benefit assessment. Furthermore, a description of what makes a robot different from a machine or a human is given. The article highlights the differences of the viewpoints and understandings of the future human-robot relationships between novice users and experts.

Communication of Emotion in Social Robots through Simple Head and Arm Movements by Jamy Li and Mark Chignell studies robot gestures. Understanding them will aid the design of robots capable of social interaction with humans. The generation and perception of a restricted form of gesture in a robot capable of simple head and arm movement is examined. Four studies are described on the effects of situational context, gesture complexity, emotional valence, and author expertise. In Study 1 and 2, four participants create gestures with corresponding emotions based on 12 scenarios. The resulting gestures are assessed by 12 judges. Their recognition of emotion is better than chance and improves when situational context is provided. In Study 3 and 4, five novices and five puppeteers create gestures conveying Ekman's six basic emotions which are shown to 12 judges. Puppetry experience improves identification rates only for the emotions of fear and disgust, possibly because of the limitations of the robot's movement.

Effect of Observing Eye Contact between a Robot and Another Person by Michihiro Shimado, Yuichiro Yoshikawa, Mana Asada, Naoki Saiwaki, and Hiroshi Ishiguro examines the potential merit offered by a triadic form of interaction. In particular, the authors investigate how one form of nonverbal interaction occurring between a robot and humans (eye-contact) can be exploited to make the robot appear more acceptable to humans. Experiments are described on groups of two humans and an android. The "subject" human is asked to communicate with a "confederate" human who has knowledge of the purpose of the experiment. The confederate's role is to gaze in such a way that the subject either observes or does not observe eye-contact between the confederate and the android. A post-interaction questionnaire reveals that the subjects' impressions toward the robot are influenced by the eye-contact between the confederate and the robot.

When Artificial Social Agents Try to Persuade People: The Role of Social Agency on the Occurrence of Psychological Reactance by Maaike Roubroeks, Jaap Ham, and Cees Midden describes how robotic agents might employ persuasion to influence people's behavior. In the study, the authors investigate the social nature of psychological reactance. Assuming that more social cues lead to more social interaction, the authors argue that this also holds for psychological reactance. The authors expect a positive relationship between the level of social agency of the source of a persuasive message and the amount of psychological reactance that the message arouses. In an online experiment, participants read an advice on how to conserve energy when using a washing machine. The results indicate that participants experience more psychological reactance when the advice is accompanied by a still picture or when the advice is accompanied by a short film clip as compared to when the advice is provided as textonly.

Reliable People Detection Using Range and Intensity Data from Multiple Layers of Laser Range Finders on a Mobile Robot by Alexander Caballo, Akihisa Ohya, and Shin'ichi Yuta describes an important task in several areas such as security, intelligent environments, and human-robot interaction. A reliable system should be able to detect static people quite adequately even in cluttered environments. The authors present a reliable approach for people detection and position estimation using multiple layers of Laser Range Finders (LRF) on a mobile robot. Each layer combines two LRF sensors to scan around the robot's surroundings. By using AdaBoost the authors create strong classifiers to detect 
body parts. Moreover, laser reflection intensity is introduced as a novel property for people detection. In the end, a thorough evaluation of the multi-layered system is provided.

Robot Vacuum Cleaner Personality and Behavior by Bram Hendriks, Bernt Meerbeek, Stella Boess, Steffen Pauws, and Marieke Sonneveld reports on the user experience of robot-vacuum-cleaner behavior. How do people want to experience this new type of cleaning appliance? Interviews are conducted to elicit a desired robot-vacuumcleaner personality. With this knowledge in mind, the behavior of a future robot vacuum cleaner is predicted. The interviewed persons give their ideas on such robots. The results indicate that people recognize the intended personality in the robot behavior. Therefore, the authors recommend using a personality model as a tool for developing a range of robot behaviors.

Humans, Animals, and Robots: A Phenomenological Approach to Human-Robot Relations by Mark Coeckelberg argues that our understanding of many human-robot relations can be enhanced by two issues. First, comparisons with human-animal relations will give us more insights. Second, a phenomenological approach will highlight the significance of how robots appear to humans. Some potential gains of the latter approach are explored by discussing the concepts alterity, diversity, and change. The author's philosophical reflections result in a perspective on human-robot relations that may guide robot design and inspire more empirical human-robot relations research. In simulation and robot experiments, the nature of deception is investigated. In the study, the author proposes a robot-like remote-control Rebo. The developed remote control has three advantages: familiarity, function awareness, and stroke manipulation.

From the seven papers we learn that understanding the artificial is a huge problem. Yet, we conjecture that currently we are already at the beginning of this track. Soon, we expect, there will be further acceleration, and after that we will cross the limits of human understanding. Then we are in the area of robot understanding. Your Editors look forward to the next stage after "the robots understanding the robot society", but they have no clue on what this might be.

Open Access This article is distributed under the terms of the Creative Commons Attribution Noncommercial License which permits any noncommercial use, distribution, and reproduction in any medium, provided the original author(s) and source are credited. 